\title{
Long-term outcomes after percutaneous revascularization of complex coronary bifurcation lesions using a dedicated self-expanding biolimus-eluting stent system
}

\author{
Andreas S. Triantafyllis ${ }^{1}$, Johan Bennett ${ }^{1}$, Efstathios Pagourelias ${ }^{1}$, Keir McCutcheon ${ }^{1}$, \\ Tom Adriaenssens ${ }^{1,2}$, Peter R. Sinnaeve ${ }^{1,2}$, Walter Desmet ${ }^{1,2}$, Christophe Dubois ${ }^{1,2}$ \\ ${ }^{1}$ Department of Cardiovascular Medicine, University Hospitals Leuven, Belgium \\ ${ }^{2}$ Department of Cardiovascular Sciences, KU Leuven, Belgium
}

\begin{abstract}
Background: To evaluate long-term clinical outcomes after treatment of complex bifurcation lesions with the AXXESS dedicated self-expanding biolimus A9-eluting bifurcation stent.

Methods: Between 2004 and 2013, 123 patients with complex bifurcation lesions were treated in a single-center with the AXXESS stent in the proximal main vessel (MV) and additional drug-eluting stents in branches when required. Median follow-up was 5 years. Primary endpoint was the rate of major adverse cardiac events (MACE). Secondary endpoints included MACE components (cardiac death, non-periprocedural clinical myocardial infarction [MI], target lesion revascularization [TLR] and definite/probable stent thrombosis [ST]) as well as all-cause death, target vessel revascularization (TVR) and non-TVR.

Results: During follow-up, 11 (8.9\%) patients experienced a MACE, of whom 2 (1.6\%) suffered cardiac death, 2 (1.6\%) had a non-periprocedural clinical MI requiring TLR, and 7 (5.7\%) underwent elective TLR. No definite/probable ST was observed. All-cause death occurred in 9 (7.3\%) patients, TVR in 11 (8.9\%) and non-TVR in 11 (8.9\%). Patients treated for left main (LM) bifurcation lesions were more likely to experience MACE than non-LM bifurcation lesions (25\% vs. $6.5 \%, p=0.04$ ).

Conclusions: Percutaneous revascularization of complex bifurcation lesions with the AXXESS stent is safe and provides excellent long-term results, especially in non-LM lesions. (Cardiol J 2018; 25, 4: 470-478)
\end{abstract}

Key words: complex bifurcation lesions, dedicated stents, AXXESS stent

\section{Introduction}

Coronary bifurcation lesions are frequently encountered in everyday clinical practice and account for $15-20 \%$ of percutaneous coronary interventions (PCI) [1]. Provisional stenting with the use of a single drug-eluting stent (DES) is the default strategy. However, in the case of significant disease affecting both the main vessel (MV) and a large side branch (SB) a two-stent strategy may be required from the outset [2]. Nevertheless, challenges remain, since tubular stent design may not conform to bifurcation anatomy and results rely on stent deformation, neocarina formation and overlapping strut layers provide full bifurcation coverage $[3,4]$. Furthermore, these technical limitations affect optimal stent apposition and re-endothelialization, which are associated with a lower procedural success rate and a higher rate of long-term adverse cardiac events such as stent restenosis

Address for correspondence: Andreas Triantafyllis, MD, PhD, Department of Cardiovascular Medicine, University Hospitals Leuven, Herestraat 49,3000 Leuven, Belgium, e-mail: andtridoc@yahoo.gr, andreas.triantafyllis@uzleuven.be 
and thrombosis [5-7]. Dedicated stents have therefore been designed to accommodate the specific anatomy of bifurcation, and to preserve branch access and support with a single stent layer [8].

The AXXESS ${ }^{\mathrm{TM}}$ stent system (Biosensors Europe SA, Morges, Switzerland) is a conical self-expanding nitinol stent, coated with a biodegradable polylactic acid (PLA) polymer eluting Biolimus A9 [9]. The present study sought to assess long-term device-oriented clinical endpoints in a large single center cohort of patients with complex coronary bifurcation lesions, including LM coronary artery bifurcations.

\section{Methods}

\section{Study population}

Patients included in the study represent pooled experience with AXXESS at the University Hospital of Leuven, Belgium, combining patients participating in different multicenter trials $[3,9$, $10,11]$ and with others treated in routine clinical practice. Between 2004 and 2013, all patients in this center with complex bifurcation lesions undergoing AXXESS stenting in the proximal MV, with or without additional stents in the branches, were included. Patients were fully informed about the procedure and collection of clinical data, and provided consent. The ethics committee of the indicated institution approved the study protocol and the latter was performed according to the principles outlined in the Declaration of Helsinki.

The study population consisted of patients with documented stable or unstable angina or a positive functional study, identified for elective PCI of a de novo native coronary bifurcation lesion. Treatment with an AXXESS device was only considered if a two-stent bifurcation treatment strategy was deemed necessary from the outset, as per the operator's judgement for optimal lesion treatment, indicating complex and diffuse disease and large myocardial territory at risk. Furthermore, eligibility required reference vessel diameter by visual estimate of $2.75-4.25 \mathrm{~mm}$ in the proximal $\mathrm{MV}$ and $>2.25 \mathrm{~mm}$ in the SB.

Two operators were involved in implantation of the AXXESS stent, one of whom performed the majority of the cases $(80 \%)$.

\section{Procedural technique}

AXXESS stent characteristics and implantation procedure have been previously described $[3,12]$. In brief, it is a self-expanding, conical-shaped nitinol stent with a strut thickness of $150 \mu \mathrm{m}$ that elutes the highly lipophilic semisynthetic sirolimus analogue Biolimus A9. The drug is emulsified into a biodegradable PLA polymer, which is absorbed over 6 to 9 months. The Biolimus A9 elution half-life is 21 days, and the drug is completely eluted by 196 days [9]. After mandatory predilatation, AXXESS stents of different sizes ( 3 and $3.5 \mathrm{~mm}$ ) and lengths (11 and $14 \mathrm{~mm}$ ) were deployed in the proximal MV, accommodating vessels from $2.75 \mathrm{~mm}$ to $3.75 \mathrm{~mm}$, with a distal flare expanding to as much as $8 \mathrm{~mm}$. A special $(4 \times 9 \mathrm{~mm})$ version of the AXXESS was used in LM bifurcation lesions, allowing for larger diameters (up to $4.75 \mathrm{~mm}$ ) and distinct bifurcation angles (flare end diameters of 8,10 and $12 \mathrm{~mm}$ ). Additional DES (sirolimus-eluting stents [SES] or biolimus-eluting stents [BES]) were implanted in case of a residual stenosis of $>30 \%$ in any segment of the bifurcation, dissection or incomplete lesion coverage. The vast majority of procedures were completed by sequential high-pressure non-compliant balloon inflations and final kissing balloon inflations.

\section{Postprocedural care and follow-up}

Dual antiplatelet therapy with acetylsalicylic acid ( $\geq 80 \mathrm{mg} /$ day) and clopidogrel (75 mg/day) was administered for at least 6 months in all patients which was extended to 12 months after the first cases. Patients were prospectively evaluated clinically in the outpatient clinic or by phone at 1-month, 1-, 3- and 5-years post-procedure.

\section{Endpoints}

The primary clinical endpoint was the rate of major adverse cardiac events (MACEs), defined as the composite of cardiac death, non-periprocedural clinical myocardial infarction (MI), target lesion revascularization (TLR) and definite/probable stent thrombosis (ST). Non-periprocedural clinical MI was defined as myocardial fraction creatine kinase (CK-MB) levels $>3 \times$ upper reference limit (URL) in combination with symptoms or electrocardiographic signs of MI occurring after the periprocedural period. Secondary endpoints included MACE components as well as all-cause death, target vessel revascularization (TVR) and non-TVR. ST was classified according to the Academic Research Consortium [13]. Reported herein are the median 5 -year results of bifurcation lesions treated with AXXESS stent in the aforementioned center.

\section{Statistical analysis}

Normality of data was tested using the Kolmogorov-Smirnov test. Summary statistics are presented as mean \pm standard deviation for contin- 
Table 1. Baseline patient characteristics.

\begin{tabular}{|c|c|c|c|c|}
\hline & $\begin{array}{l}\text { All patients } \\
(n=123)\end{array}$ & $\begin{array}{l}\text { No MACEs } \\
(n=112)\end{array}$ & $\begin{array}{l}\text { MACEs } \\
(n=11)\end{array}$ & $\mathbf{P}$ \\
\hline \multicolumn{5}{|l|}{ Demographics: } \\
\hline Age [years] & $64.7 \pm 10.2$ & $64.6 \pm 10.3$ & $65.4 \pm 9.2$ & 0.82 \\
\hline Male (gender) & $91(74 \%)$ & $82(73.2 \%)$ & $9(81.8 \%)$ & 0.81 \\
\hline Body mass index $\left[\mathrm{kg} / \mathrm{m}^{2}\right]$ & $27.3 \pm 3.6$ & $27.4 \pm 3.7$ & $26.6 \pm 2.6$ & 0.53 \\
\hline \multicolumn{5}{|l|}{ Cardiac risk factors: } \\
\hline Diabetes mellitus & $25(20.3 \%)$ & $23(20.5 \%)$ & $2(18.2 \%)$ & 1.00 \\
\hline Arterial hypertension & $75(61 \%)$ & $68(60.7 \%)$ & $7(63.6 \%)$ & 1.00 \\
\hline Hyperlipidemia & $105(85.4 \%)$ & $94(83.9 \%)$ & $11(100 \%)$ & 0.37 \\
\hline Smoking: & & & & 0.14 \\
\hline Current & $27(22 \%)$ & $27(24.1 \%)$ & $0(0 \%)$ & \\
\hline Former & $44(35.8 \%)$ & $38(33.9 \%)$ & $6(54.5 \%)$ & \\
\hline Never & $52(42.3 \%)$ & $47(42 \%)$ & $5(45.5 \%)$ & \\
\hline Family history of CAD & $62(50.4 \%)$ & $55(49.1 \%)$ & $7(63.6 \%)$ & 0.53 \\
\hline Previous myocardial infarction & $33(26.8 \%)$ & $32(28.6 \%)$ & $1(9.1 \%)$ & 0.29 \\
\hline Previous PCl & $51(41.5 \%)$ & $45(40.2 \%)$ & $6(54.5 \%)$ & 0.52 \\
\hline Previous CABG & $7(5.7 \%)$ & $5(4.5 \%)$ & $2(18.2 \%)$ & 0.12 \\
\hline \multicolumn{5}{|l|}{ Chronic kidney disease } \\
\hline Estimated GFR $<60 \mathrm{~mL} / \mathrm{min} / 1.73 \mathrm{~m}^{2}$ & $15(12.2 \%)$ & $14(12.5 \%)$ & $1(9.1 \%)$ & 1.00 \\
\hline Heart failure (> NYHA class II) & $2(1.6 \%)$ & $2(1.8 \%)$ & $0(0 \%)$ & 1.00 \\
\hline Peripheral vascular disease & $19(15.4 \%)$ & $17(15.2 \%)$ & $2(18.2)$ & 0.68 \\
\hline Previous cerebrovascular disease & $9(7.3 \%)$ & $8(7.1 \%)$ & $1(9.1 \%)$ & 0.58 \\
\hline Clinical presentation: & & & & 0.29 \\
\hline Stable (angina) & $89(72.4 \%)$ & $83(74.1 \%)$ & $6(54.5 \%)$ & \\
\hline Unstable (angina) & $30(24.4 \%)$ & $26(23.2 \%)$ & $4(36.4 \%)$ & \\
\hline Silent (ischemia) & $4(3.3 \%)$ & $3(2.7 \%)$ & $1(9.1 \%)$ & \\
\hline Left ventricular ejection fraction [\%] & $60.9 \pm 7.4$ & $61 \pm 7.5$ & $60.2 \pm 7.1$ & 0.78 \\
\hline
\end{tabular}

Values are mean \pm standard deviation or number (percentage).

CABG - coronary artery bypass graft; CAD — coronary artery disease; GFR — glomerular filtration rate; MACEs — major adverse cardiac events consisting of cardiac death, non-periprocedural clinical myocardial infarction, target lesion revascularization and definite/probable stent thrombosis; NYHA - New York Heart Association; PCl — percutaneous coronary intervention

uous variables and as absolute numbers (percentages) for categorical data. Comparisons between the groups of patients with and without MACEs were performed using the two-tailed Student $t$ test for continuous variables; comparison of categorical variables was assessed by the $\chi^{2}$ test. The variables that were found to be statistically different between the two groups were then fitted to univariate and multiple logistic regression models, in order to depict the best predicting factors of MACEs in the documented population. Estimates of odds ratios (ORs) and 95\% confidence intervals (CIs) for each variable are presented. To evaluate event-free survival rates for each event, the Kaplan-Meier method was applied and 1-, 3- and 5-year eventfree survival with $95 \%$ CI were reported and sur- vival curves constructed. Comparison of survival curves was performed based on a log rank test. All statistical analyses were performed using SPSS statistics software (v 20.0, IBM, Chicago, Illinois) and Medcalc software (version 15.2.2, Ostend, Belgium). A two-sided p-value $<0.05$, was considered statistically significant for all tests.

\section{Results}

The baseline characteristics of 123 patients in whom AXXESS stent implantation was intended are summarized in Table 1 . The majority were male (74\%) with a mean age of 64.7 years and multiple risk factors for ischemic heart disease. Most patients $(72.4 \%)$ presented with stable angina. 
Lesion and procedural characteristics are summarized in Table 2. Most lesions (78\%) were true bifurcation lesions (I-I-I, I-0-I and 0-I-I according to the Medina system) [14], most often involving the left anterior descending/diagonal (LAD/D) bifurcation $(73.2 \%)$, while LM bifurcation accounted for $13 \%$ only.

Overall device success, defined as deployment of AXXESS without system failure or devicerelated complication, was achieved in 118 (95.9\%) patients. In 2 cases, the AXXESS stent could not be advanced and delivered in the bifurcation due to anatomical restrictions and in 1 patient the stent was lost in the iliac artery. Two others received an AXXESS stent, however in a too proximal position. In these cases, the target bifurcation was successfully treated with tubular DES only. Overall, lesion success (residual stenosis $<50 \%$ in any bifurcation segment) was obtained in 122 (99.2\%) patients.

Satisfactory bifurcation treatment with an AXXESS stent alone was achieved in 12/118 patients (10.2\%). After AXXESS deployment, additional DES were implanted in distal MV



Figure 1. Distribution of stent implantation scenarios. AXXESS stents were implanted in the proximal main vessel (MV), with or without additional tubular drugeluting stents in the distal MV or side-branch (SB).

and/or SB in $106 / 118$ patients $(89.8 \%)$. The distribution of these different scenarios is illustrated in Figure 1. Finally, in 12/118 patients

Table 2. Procedural characteristics.

\begin{tabular}{|c|c|c|c|c|}
\hline & $\begin{array}{l}\text { All patients } \\
(\mathrm{n}=123)\end{array}$ & $\begin{array}{l}\text { No MACEs } \\
(n=112)\end{array}$ & $\begin{array}{l}\text { MACEs } \\
(n=11)\end{array}$ & $\mathbf{P}$ \\
\hline \multicolumn{5}{|l|}{ Target lesion (main branch): } \\
\hline Left main & $16(13 \%)$ & $12(10.8 \%)$ & $4(36.4 \%)$ & 0.03 \\
\hline Left anterior descending & $90(73.2 \%)$ & $86(76.8 \%)$ & $4(36.4 \%)$ & \\
\hline Circumflex & $13(10.5 \%)$ & $11(9.9 \%)$ & $2(18.2 \%)$ & \\
\hline Right coronary artery & $4(3.3 \%)$ & $3(2.7 \%)$ & $1(9.1 \%)$ & \\
\hline Medina classification: & & & & 0.88 \\
\hline $1-1-1$ & $75(61 \%)$ & $68(60.7 \%)$ & $7(63.6 \%)$ & \\
\hline $1-1-0$ & $14(11.4 \%)$ & $13(11.6 \%)$ & $1(9.1 \%)$ & \\
\hline $1-0-1$ & $9(7.3 \%)$ & $8(7.1 \%)$ & $1(9.1 \%)$ & \\
\hline $1-0-0$ & $7(5.7 \%)$ & $6(5.4 \%)$ & $1(9.1 \%)$ & \\
\hline $0-1-1$ & $12(9.8 \%)$ & $12(10.7 \%)$ & $0(0 \%)$ & \\
\hline $0-1-0$ & $1(0.8 \%)$ & $1(0.9 \%)$ & $0(0 \%)$ & \\
\hline $0-0-1$ & $5(4.1 \%)$ & $4(3.6 \%)$ & $1(9.1 \%)$ & \\
\hline \multicolumn{5}{|l|}{ Calcification: } \\
\hline Proximal main vessel & $29(23.6 \%)$ & $27(24.1 \%)$ & $2(18.2 \%)$ & 1.00 \\
\hline Distal main vessel & $29(23.6 \%)$ & $27(24.1 \%)$ & $2(18.2 \%)$ & 1.00 \\
\hline Side branch & $23(18.7 \%)$ & $21(18.8 \%)$ & $2(18.2 \%)$ & 1.00 \\
\hline Total number of stents/patients & $2.7 \pm 07$ & $2.7 \pm 0.8$ & $2.7 \pm 0.6$ & 0.84 \\
\hline Total stent length/patient [mm] & $37.1 \pm 14.4$ & $37.4 \pm 14.8$ & $33.8 \pm 8.7$ & 0.25 \\
\hline Patients with additional stents & $12(9.8 \%)$ & $12(10.7 \%)$ & $0(0 \%)$ & 0.60 \\
\hline Device success & $118(95.9 \%)$ & $107(95.5 \%)$ & $11(100 \%)$ & 1.00 \\
\hline Final kissing balloon & $112(91.1 \%)$ & $102(91.1 \%)$ & $10(90.9 \%)$ & 1.00 \\
\hline Lesion success & $122(99.2 \%)$ & $111(99.1 \%)$ & $11(100 \%)$ & 1.00 \\
\hline
\end{tabular}

Values are mean \pm standard deviation or number (percentage). MACEs - major adverse cardiac events consisting cardiac death, non-periprocedural clinical myocardial infarction, target lesion revascularization and definite/probable stent thrombosis 


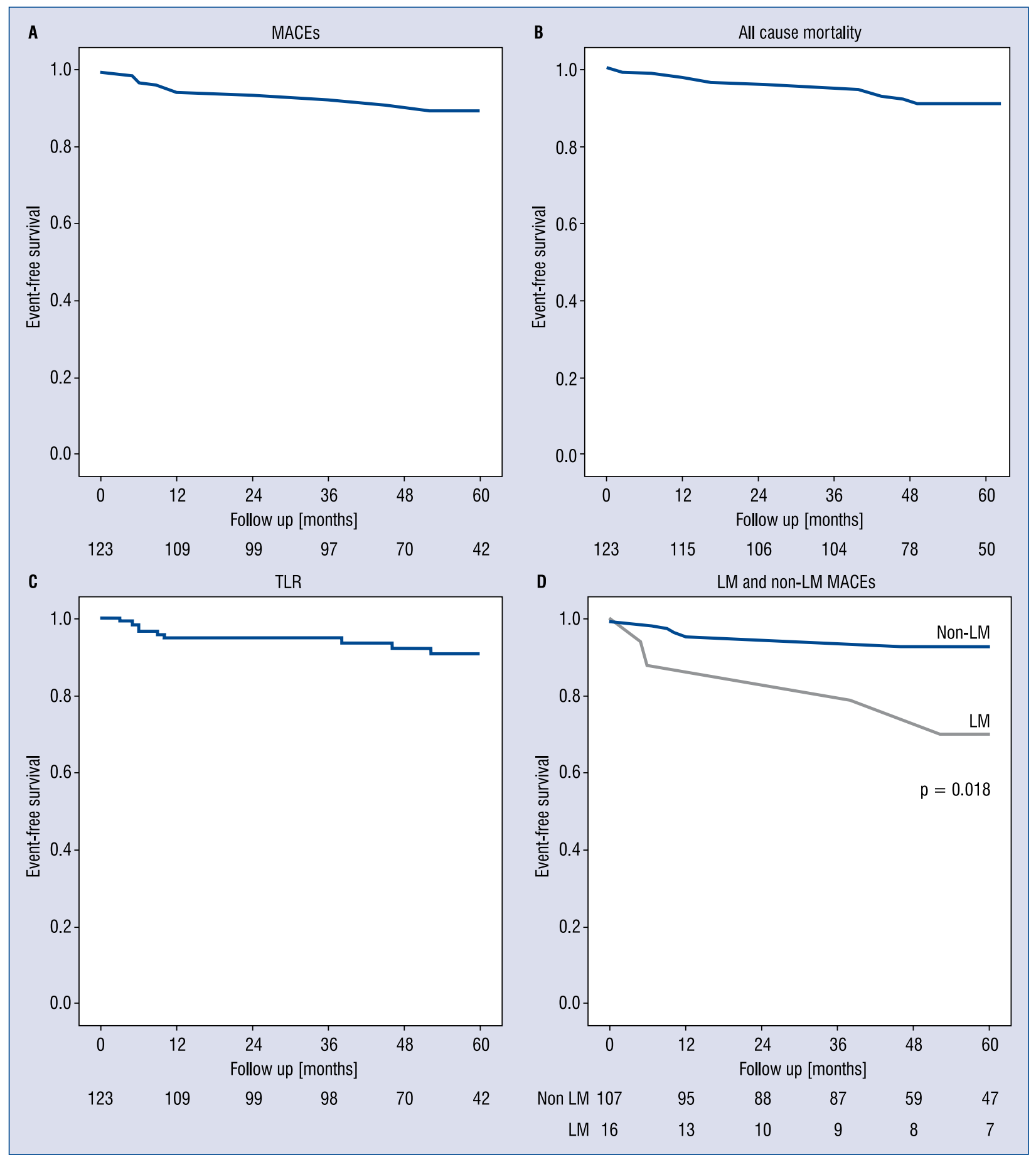

Figure 2. Event-free survival curves. Kaplan-Meier event-free survival curves are presented for major adverse cardiac events (MACEs; A), all-cause death (B), target lesion revascularization (TLR; C); D. MACE-free survival after left main (LM) and non-LM intervention are compared using log rank.

(10.2\%) further additional DES were implanted due to incomplete lesion coverage or proximal/distal edge dissections.

All patients underwent clinical follow-up at 1 and 3 years, and $69.1 \%(n=85)$ could be assessed at 5 years. No patients were lost to follow-up. Median time from inclusion up to latest follow-up was 60 months. Kaplan-Meier event-free survival curves for MACE, all-cause death and TLR are presented in Figure 2A-C. 
Table 3. Event-free survival during follow-up.

\begin{tabular}{|c|c|c|c|c|}
\hline & $\begin{array}{l}\text { All patients } \\
(\mathrm{n}=123)\end{array}$ & $\begin{array}{c}\text { 1-year } \\
(\%)\end{array}$ & $\begin{array}{c}\text { 3-years } \\
(\%)\end{array}$ & $\begin{array}{c}\text { 5-years } \\
(\%)\end{array}$ \\
\hline Non-periprocedural MI & $2(1.6 \%)$ & & & \\
\hline All cause mortality: & $9(7.3 \%)$ & $98.3(95.9-99.9)$ & 95.6 (91.9-99.3) & $90.9(85.2-96.6)$ \\
\hline Cardiac death & $2(1.6 \%)$ & & & \\
\hline Non-cardiac death & $7(5.7 \%)$ & & & \\
\hline Definite/probable ST & 0 & & & \\
\hline Target lesion revascularization & $9(7.3 \%)$ & $94.9(91-98.8)$ & $94.9(91-98.8)$ & $91(85.3-96.7)$ \\
\hline Target vessel revascularization & $11(8.9 \%)$ & & & \\
\hline Non-target vessel revascularization & $11(8.9 \%)$ & $94.9(91-98.8)$ & 90.2 (84.7-95.7) & $90.2(84.7-95.7)$ \\
\hline MACEs & $11(8.9 \%)$ & 94 (89.7-98.3) & 93.1 (88.4-97.8) & $89.3(83.2-95.4)$ \\
\hline
\end{tabular}

Values are number (percentage). Event free survival data represent percentages and in parentheses $95 \%$ confidence intervals for the most important endpoints. MACE - major adverse cardiac events consisting cardiac death, non-periprocedural clinical myocardial infarction, target lesion revascularization and definite/probable stent thrombosis; MI - myocardial infarction; ST — stent trombosis

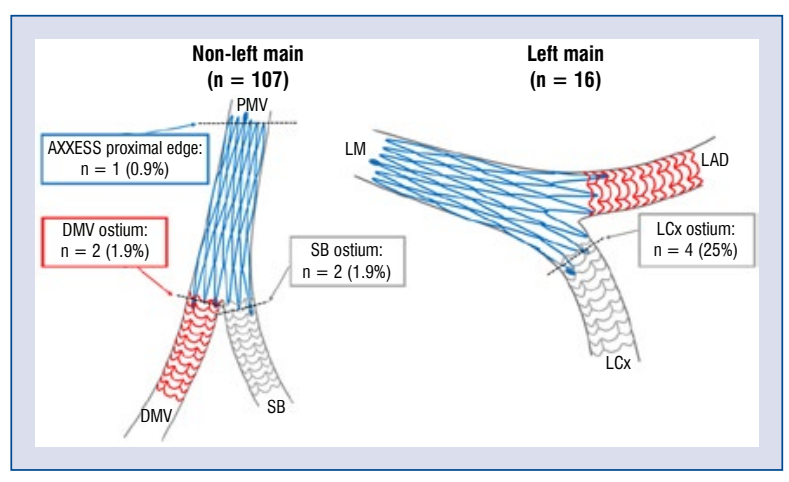

Figure 3. Distribution of restenosis triggering target lesion revascularization (TLR). Among 5 restenoses leading to TLR in non-left main (LM) bifurcations (left panel), one was located at the proximal edge of the AXXESS stent, and 4 affected the ostium of the branches (twice the distal main vessel [MV] and twice the side-branch [SB]). In LM bifurcations, restenosis at the ostium of the circumflex artery led to TLR in 4 cases; DMV - distal main vessel; LAD - left anterior descending; LCx circumflex; PMV - proximal main vessel.

During follow-up, $11(8.9 \%)$ patients suffered MACE, of whom $2(1.6 \%)$ patients with cardiac death, $2(1.6 \%)$ with non-periprocedural clinical MI requiring TLR, and 7 (5.7\%) others underwent elective TLR. There was 1 sudden cardiac death 3 years after the index procedure, accounting for one possible ST. All-cause death occurred in $9(7.3 \%)$ and TLR was performed in $7.3 \%$ of the patients. Finally, TVR occurred in $11(8.9 \%)$ and non-TVR in $11(8.9 \%)$ patients. A full list of events and eventfree survival are illustrated in Table 3.
Affected bifurcation segments and disease pattern triggering TLR are shown in Figure 3. In most cases, restenosis was due to failure of the additional DES in the distal branches, while the AXXESS segment was rarely involved. Patients treated for LM bifurcation lesions were more likely to experience MACE when compared with non-LM bifurcation lesions ( $25 \%$ vs. $6.5 \%, \mathrm{p}=0.04)$. LM bifurcation lesions were also an independent predictor for TLR (HR 5.36, 95\% CI 1.4-19.9, p = 0.02). Kaplan-Meier event-free survival curves for LM vs. non-LM bifurcation lesions are presented in Figure 2D. Furthermore, stent length of MV, SB or total bifurcation stent length did not appear to affect MACE rate (data not shown).

\section{Discussion}

The present study assessed long-term clinical outcomes after percutaneous revascularization of complex coronary bifurcation lesions with the AXXESS stent, a second generation DES specifically designed for bifurcation lesions. In this large cohort of patients with predominantly true bifurcation lesions, highly successful AXXESS implantation and accurate stent positioning, with or without the use of additional DES in the distal branches, resulted in acute lesion success in all but 1 patient. Five-year clinical follow-up confirmed favorable outcomes, with single digit MACE rates (8.9\%), cardiac death-, MI- and TLR rates as low as $1.6 \%, 1.6 \%$ and $7.3 \%$, respectively, and no definite/ /probable ST. While most patients were treated for lesions in $\mathrm{LAD} / \mathrm{D}$ bifurcation, a small number of patients undergoing treatment of LM bifurca- 
tion were 4 times more likely to suffer an MI and 5 times more likely to undergo TLR during follow-up.

By design, dedicated bifurcation devices offer solutions against the limitations of tubular stents by conforming to bifurcation anatomy and avoiding stent deformation and overlap. Theoretically, they represent a valid alternative to the ongoing controversy of single versus double tubular stent strategies to be used in a bifurcation scenario [15-17]. However, implantation of dedicated bifurcation stents is technically demanding and the learning curve relating to patient/lesion selection and technical implementation, both hamper breakthrough of the technique in daily practice as well as evaluation of its true value. Additionally, the widely used Medina bifurcation classification does not take into account calcification or bifurcation angle, which preferably should remain $<70^{\circ}$ with the AXXESS device, making patient/lesion selection less straightforward. Moreover, brilliant dedicated stent concepts struggle with limitations inherent to the smaller scale and the often heterogeneous character of initial clinical experiences, hereby reinforcing the unfavorable impact of the learning curve. Finally, niche products have a hard time competing with workhorse products in following the pace of innovation.

The present report offers solid and long-term data in a sizeable group of patients with complex bifurcation disease treated with the AXXESS stent over a period of 10 years. The initial experience was gathered during the heydays of first generation sirolimus and paclitaxel-eluting products, reinforcing the innovative value of this dedicated device in terms of bioresorbable polymer coating and alternative limus-elution. While the design of the AXXESS has not changed over time, its modular concept allows for combinations with the latest generation DES, or even fully bioresorbable scaffolds [18], while luminal gain at the level of proximal MV remains unprecedented [3]. The strength of the present registry is in the dedication of a single team in the treatment of complex bifurcation lesions with a dedicated device, completeness of clinical follow-up and favorable long-term results.

In this non-randomized study of a dedicated bifurcation device, there was a deliberate choice to focus on a device-oriented composite of cardiac death, MI and TLR as a MACE endpoint, in order to reflect long-term clinical device performance. It was decided not to include periprocedural MI in the composite endpoint to avoid confusion related to continuously changing MI definitions throughout the period of inclusion. In addition, due to heteroge- neity of troponin assays and enzymatic techniques during the span of the study, CK-MB was used as a marker of myocardial necrosis. Finally, evaluation of individual endpoints appears to be the most trustworthy method of comparing outcomes as with a large assortment of available bifurcation studies.

Notwithstanding favorable outcomes with AXXESS in a larger multicenter experience in the AXXESS Plus and DIVERGE trial, 5-year followup in the present experience resulted numerically in even lower rates of cardiac death $(1.6 \%$ vs. $3.3 \%)$, non-periprocedural clinical MI (1.6\% vs. $5.5 \%)$, ischemia-driven TLR $(7.3 \%$ vs. $11.5 \%)$ and no definite/probable ST vs. 3\% [19]. Of note, most events in this cohort occurred in patients with LM bifurcation lesions, while disease in this bifurcation segment was an exclusion criterion in the aforementioned multicenter trials.

The present results also compare favorably with patients treated with tubular BES in bifurcation segments in the all-comers LEADERS trial, using an identical polymer and drug concept as in AXXESS, and where clearly higher rates of cardiac death $(9.3 \%)$, TLR (12\%) and definite/probable ST $(3.1 \%)$ were reported at 5 years [20]. Similarly, AXXESS in the present experience proved to be as safe and efficacious as later-generation thin-strut everolimus-eluting stents in the PLATINUM study treating non-bifurcation lesions, with similar rates of cardiac death (3.1\%), TLR (5.7\%) and definite/ /probable ST $(0.8 \%)$ at 5 years [21].

Among studies specifically designed to elucidate the controversy on single vs. double DES approach in bifurcation scenarios, pooled results of the NORDIC I and BBC ONE studies showed an all-cause death rate at 5 years of $6.2 \%$, within the range of what is reported herein with AXXESS [22]. More specifically, in NORDIC I, even in the single stent group, a numerically higher rate of cardiac death (2.5\%), non-periprocedural MI (4\%), TLR $(11.3 \%)$ and definite ST (3.0\%) was seen [23]. Of note, these studies utilized first generation SES and paclitaxel-eluting stent, as was the case in the majority of cases in the present AXXESS experience, whenever additional DES were considered appropriate. In contrast, the present experience included more true bifurcation lesions and a significantly longer cumulative stent length.

In addition, in the present study and within limitations of clinical follow-up, it was undertaken to elucidate modes of device failure and found that it rarely involved the AXXESS segment. Only 1 patient had a TLR related with proximal edge restenosis of the AXXESS. All other TLRs oc- 
curred in the ostial branch segments where DES were used to cover the bifurcation, the majority of which were first generation DES (68.5\%, cypher SES), which may have influenced the restenosis process. The low rates of cardiac death observed would suggest that device failure seldom leads to catastrophic events.

In the present experience with AXXESS, treatment of LM bifurcation appeared to be an independent predictor of TLR and MACE. Potential contributors to this worse outcome are early operator experience and distinct behaviour of the AXXESS stent in a different anatomic environment. Indeed, most LM treatments took place at the early stage of present experience, and most events were concentrated in the initial AXXESS cohort (data not shown). In contrast, it remains unclear to what extent specific anatomy of LM bifurcation with wider angles and near-orthogonal take-off of the circumflex contributed to higher rates of target lesion failure with AXXESS [24]. Indeed, the specifically adapted and trumpet-shaped design of the LM stent, including a wider span at the distal end, was expected to favour LM bifurcation outcomes, due to its carina-sparing properties, the avoidance of neocarina formation and extensive double stent layers, and its conformance to bifurcation anatomy. However, despite its specific wide-angle design, close apposition of the stent to the vessel at the ostium of the circumflex artery may have been insufficient, as has been seen with more traditional double stent approaches in the complex threedimensional structure of the LM [25]. Conceptually, though, a modular approach of LM bifurcation reconstruction remains attractive, and is probably one of the few settings in which operators are inclined to use a multiple stent strategy, provided normal anatomy and flow can be restored. Of note, further development and marketing of the specific LM AXXESS has currently been abandoned.

\section{Limitations of the study}

The present study has several limitations. Sample size of this single center experience remains relatively limited in assessing clinical outcomes, especially in the LM group. Secondly, most DES used in conjunction with AXXESS during a long inclusion period no longer represent contemporary PCI practice. However, while some of these DES may have negatively influenced overall outcome results, early implementation of second generation DES technology with AXXESS proved to remain a valid option for bifurcation PCI. Finally, no formal comparisons with other bifurca- tion techniques were performed in this study, but recent evidence revealed the value of AXXESS as compared with a culotte technique with latest generation DES [3].

\section{Conclusions}

The results of this study suggest that percutaneous revascularization of complex coronary bifurcation lesions with dedicated AXXESS bifurcation stent, with or without additional DES in the branches, provides a safe and attractive modular reconstruction of the bifurcation with excellent 5 -year results in terms of survival and MACE. The AXXESS stent can especially be considered in large non-LM bifurcations, mainly involving the $\mathrm{LAD} / \mathrm{D}$ anatomy, with an appropriate angle when more than a provisional approach is anticipated.

\section{Conflict of interest: None declared}

\section{References}

1. Stankovic G, Lassen JF, Hildick-Smith D, et al. The EuroIntervention coronary bifurcation treatment supplement. EuroIntervention. 2015; 11 Suppl V: V9-11, doi: 10.4244/EIJV11SVA2, indexed in Pubmed: 25983181.

2. Behan MW, Holm NR, Curzen NP, et al. Simple or complex stenting for bifurcation coronary lesions: a patient-level pooledanalysis of the Nordic Bifurcation Study and the British Bifurcation Coronary Study. Circ Cardiovasc Interv. 2011; 4(1): 57-64, doi: 10.1161/CIRCINTERVENTIONS.110.958512, indexed in Pubmed: 21205942.

3. Dubois C, Bennett J, Dens J, et al. COmplex coronary Bifurcation lesions: RAndomized comparison of a strategy using a dedicated self-expanding biolimus-eluting stent versus a culotte strategy using everolimus-eluting stents: primary results of the COBRA trial. EuroIntervention. 2016; 11(13): 1457-1467, doi: 10.4244/ EIJY15M05_02, indexed in Pubmed: 25947278.

4. Iakovou I, Ge L, Colombo A. Contemporary stent treatment of coronary bifurcations. J Am Coll Cardiol. 2005; 46(8): 1446-1455, doi: 10.1016/j.jacc.2005.05.080, indexed in Pubmed: 16226167.

5. Farb A, Burke AP, Kolodgie FD, et al. Pathological mechanisms of fatal late coronary stent thrombosis in humans. Circulation. 2003; 108(14): 1701-1706, doi: 10.1161/01.CIR.0000091115.05480.B0, indexed in Pubmed: 14504181.

6. Finn AV, Joner M, Nakazawa G, et al. Pathological correlates of late drug-eluting stent thrombosis: strut coverage as a marker of endothelialization. Circulation. 2007; 115(18): 2435-2441, doi: 10.1161/CIRCULATIONAHA.107.693739, indexed in Pubmed: 17438147 .

7. Iakovou I, Schmidt T, Bonizzoni E, et al. Incidence, predictors, and outcome of thrombosis after successful implantation of drugeluting stents. JAMA. 2005; 293(17): 2126-2130, doi: 10.1001/ jama.293.17.2126, indexed in Pubmed: 15870416.

8. Lefèvre T, Chevalier B, Louvard Y. Is there a need for dedicated bifurcation devices? EuroIntervention. 2010; 6 Suppl J: J123-J129, doi: 10.4244/EIJV6SUPJA20, indexed in Pubmed: 21930476. 
9. Buysschaert I, Dubois CL, Dens J, et al. Three-year clinical results of the Axxess Biolimus A9 eluting bifurcation stent system: the DIVERGE study. EuroIntervention. 2013; 9(5): 573-581, doi: 10.4244/EIJV9I5A93, indexed in Pubmed: 24058075.

10. Hasegawa T, Ako J, Koo BK, et al. Analysis of left main coronary artery bifurcation lesions treated with biolimus-eluting DEVAX AXXESS plus nitinol self-expanding stent: intravascular ultrasound results of the AXXENT trial. Catheter Cardiovasc Interv. 2009; 73(1): 34-41, doi: 10.1002/ccd.21765, indexed in Pubmed: 19089934.

11. Miyazawa A, Ako J, Hassan A, et al. Analysis of bifurcation lesions treated with novel drug-eluting dedicated bifurcation stent system: intravascular ultrasound results of the AXXESS PLUS trial. Catheter Cardiovasc Interv. 2007; 70(7): 952-957, doi: 10.1002/ccd.21269, indexed in Pubmed: 18044777.

12. Dubois CL, Wijns W. The AXXESS ${ }^{\mathrm{TM}}$ self-expanding biolimus $\mathrm{A} 9^{\mathrm{TM}}$ eluting stent system for coronary bifurcation lesions. EuroIntervention. 2010; 6 Suppl J: J130-J134, doi: 10.4244/EIJV6SUPJA21, indexed in Pubmed: 21930477.

13. Cutlip DE, Windecker S, Mehran R, et al. Academic Research Consortium. Clinical end points in coronary stent trials: a case for standardized definitions. Circulation. 2007; 115(17): 2344-2351, doi: 10.1161/CIRCULATIONAHA.106.685313, indexed in Pubmed: 17470709.

14. Medina A, Lezo JS, Pan M. A new classification of coronary bifurcation lesions. Rev Esp Cardiol. 2006; 59(2): 183, doi: 10.1016/ s1885-5857(06)60130-8.

15. Bennett J, Dubois C. Coronary bifurcation lesions: is less more? J Thorac Dis. 2016; 8(10): E1351-E1354, doi: 10.21037/ jtd.2016.10.32, indexed in Pubmed: 27867625.

16. Chen L, Fan L, Luo Y, et al. Ex vivo mono-ring technique simplifies culotte stenting for treatment of true bifurcation lesions: Insights from bench testing and clinical application. Cardiol J. 2016; 23(6): 673-684, doi: 10.5603/CJ.a2016.0054, indexed in Pubmed: 27515483.

17. Kern A, Gil RJ, Bojko K, et al. The approach to coronary bifurcation treatment and its outcomes in Poland: The single center experience. Cardiol J. 2017; 24(6): 589-596, doi: 10.5603/ CJ.a2017.0057, indexed in Pubmed: 28541601.

18. Bennett J, Adriaenssens T, Desmet W, et al. Complex bifurcation lesions: Randomized comparison of a fully bioresorbable modified $t$ stenting strategy versus bifurcation reconstruction with a dedicated self-expanding stent in combination with bioresorb- able scaffolds, an OCT study: Rationale and design of the COBRA II trial. Catheter Cardiovasc Interv. 2016; 88(6): 843-853, doi: 10.1002/ccd.26571, indexed in Pubmed: 27184586.

19. Verheye S, Buysschaert I, Grube E. Impact of side branch stenting on five-year long-term clinical outcome with the bifurcationdedicated Axxess Biolimus A9-eluting stent system. EuroIntervention. 2015; 11(8): 860-867, doi: 10.4244/EIJV11I8A176, indexed in Pubmed: 26696454.

20. Grundeken MJ, Wykrzykowska JJ, Ishibashi Y, et al. First generation versus second generation drug-eluting stents for the treatment of bifurcations: 5-year follow-up of the LEADERS all-comers randomized trial. Catheter Cardiovasc Interv. 2016; 87(7): E248-E260, doi: 10.1002/ccd.26344, indexed in Pubmed: 26649651.

21. Stone G, Teirstein P, Meredith I, et al. Final five-year results of the platinum randomized trial comparing platinum chromium promus element and cobalt chromium promus/xience v everolimus-eluting stents in workhorse lesions. J Am Coll Cardiol. 2015; 65(10): A1733, doi: 10.1016/s0735-1097(15)61733-8.

22. Behan MW, Holm NR, de Belder AJ, et al. Coronary bifurcation lesions treated with simple or complex stenting: 5 -year survival from patient-level pooled analysis of the Nordic Bifurcation Study and the British Bifurcation Coronary Study. Eur Heart J. 2016; 37(24): 1923-1928, doi: 10.1093/eurheartj/ehw170, indexed in Pubmed: 27161619.

23. Maeng M, Holm NR, Erglis A, et al. Nordic-Baltic Percutaneous Coronary Intervention Study Group. Long-term results after simple versus complex stenting of coronary artery bifurcation lesions: Nordic Bifurcation Study 5-year follow-up results. J Am Coll Cardiol. 2013; 62(1): 30-34, doi: 10.1016/j.jacc.2013.04.015, indexed in Pubmed: 23644088.

24. Kang SJ, Mintz GS, Kim WJ, et al. Changes in left main bifurcation geometry after a single-stent crossover technique: an intravascular ultrasound study using direct imaging of both the left anterior descending and the left circumflex coronary arteries before and after intervention. Circ Cardiovasc Interv. 2011; 4(4): 355-361, doi: 10.1161/CIRCINTERVENTIONS.110.961045, indexed in Pubmed: 21712525.

25. Murasato Y, Horiuchi M, Otsuji Y. Three-dimensional modeling of double-stent techniques at the left main coronary artery bifurcation using micro-focus X-ray computed tomography. Catheter Cardiovasc Interv. 2007; 70(2): 211-220, doi: 10.1002/ccd.21133, indexed in Pubmed: 17421017. 